\title{
The abilities of the aphid parasitoids Aphidius ervi Haliday and A. rhopalo- siphi De Stefani Perez (Hymenoptera: Braconidae) to transfer between different known host species and the implications for the use of alternative hosts in pest control strategies
}

\author{
W. Powell and A. F. Wright \\ AFRC, Institute of Arable Crops Research, Rothamsted Experimental Station, Harpenden, Herts, \\ $A L 52 J Q, U K$
}

\begin{abstract}
A series of host-transfer trials using both laboratory-cultured and fieldcollected individuals of the aphid parasitoids Aphidius ervi Haliday and $A$. rhopalosiphi De Stefani Perez were done in order to clarify inconsistent results from several previous studies. A. ervi cultured on Acyrthosiphon pisum (Harris) produced very few mummies when confined with Microlophium carnosum (Buckton), whereas those cultured on $M$. carnosum produced as many mummies on $A$. pisum as they did on their original host. Mummy production was correlated with the attack rate of adult parasitoids on potential hosts. The production of mummies on $M$. carnosum by parasitoids reared on $A$. pisum was often greatly improved if their male parent had been reared on $M$. carnosum, suggesting that genotype strongly influences host preference. Aphidius rhopalosiphi from laboratory cultures produced significantly more mummies on Metopolophium dirhodum (Walker) than on Sitobion avenae $(\mathrm{F}$.) regardless of their original host, but this preference was not shown by parasitoids from field populations. It is concluded that inadvertent selection occurs in laboratory cultures of aphid parasitoids as a result of low founder numbers, genetic drift and genetic bottlenecks and that this can strongly influence experimental results in biology and behaviour studies, which has important implications for biological control workers.
\end{abstract}

\section{Introduction}

The impact of insect parasitoids on crop pest populations may be enhanced by the availability of alternative hosts in or around the crop (Powell, 1986). It has been suggested that alternative hosts can help to improve synchrony between parasitoids and their pest hosts, improve parasitoid distribution and reduce intraspecific competition in the parasitoid population (van den Bosch \& Telford, 1964).

The usefulness of wild plants within arable farmland in providing alternative hosts for parasitoids attacking aphids on nearby crops has been suggested by Starý (1986) and Starý \& Němec (1986). Both Perrin (1975) and Starý (1983a) have proposed that the aphid Microlophium carnosum (Buckton) on its host-plant, the perennial stinging nettle (Urtica dioica), could act as a reservoir host for the parasitoid Aphidius ervi Haliday, which also attacks pest species such as the pea aphid, Acyrthosiphon pisum (Harris), and the cereal aphid, Sitobion avenae (F.). Aphidius ervi has become one of the most widely used 
parasitoids in aphid control programmes, particularly against Acyrthosiphon pisum and A. kondoi Shinji on lucerne.

However, the value of an alternative host will depend upon the facility with which parasitoids will switch between it and hosts on nearby crops. In laboratory trials, Aphidius ervi reared on Acyrthosiphon pisum would not attack the nettle aphid, $M$. carnosum, and gave reduced rates of mummy production when switched to $S$. avenae (Cameron et al., 1984). Similarly, Pungerl (1984) failed to induce Aphidius ervi from $M$. carnosum to transfer to $S$. avenae, and although it did transfer to Acyrthosiphon pisum, very few mummies were produced. Reduced mummy production when Aphidius ervi was switched from M. carnosum to Acyrthosiphon pisum was also noted by Starý et al., (1985). In an effort to shed more light on this problem, a more extensive series of host-transfer trials, using both laboratory and field populations of Aphidius ervi, was carried out.

It is known that aphid parasitoids respond to odours from the food-plants of their hosts in olfactometer tests (Read et al., 1970; Powell \& Zhang, 1983) and that kairomones from these food-plants can influence the behaviour of the parasitoids (Powell, 1986; Nordlund, 1987). Therefore, as the main host species of $A$. ervi occur on very different food-plants, a complementary series of trials was done using a closely related species, $A$. rhopalosiphi De Stefani Perez, transferring between the cereal aphids $S$. avenae and Metopolophium dirhodum (Walker) on the same food-plant, wheat.

\section{Materials and methods}

\section{Laboratory cultures}

Laboratory cultures of $A$. ervi were established on both Acyrthosiphon pisum and Microlophium carnosum and of Aphidius rhopalosiphi on both S. avenae and Metopolophium dirhodum, using 10-20 parasitoids reared from field-collected aphids of each appropriate host species. All aphids were collected from Rothamsted Farm, Acyrthosiphon pisum from lucerne, Microlophium carnosum from nettles in field margins and the two cereal aphids from winter wheat crops. To maintain the parasitoids in culture, all host aphids were reared on potted plants in a glasshouse, $A$. pisum on broad bean, Vicia faba, seedlings, $M$. carnosum on nettle cuttings and $S$. avenae and Metopolophium dirhodum on winter wheat seedlings. All cultures were maintained in wooden-framed cages with glass doors and fine-mesh, nylon-netting sides, and the parasitoid cultures were kept in a constant environment room at a temperature of $20 \pm 1^{\circ} \mathrm{C}$ with an $18-\mathrm{h}$ daylength.

For convenience, parasitoids reared from $A$. pisum and from Microlophium carnosum will be referred to as Aphidius ervi-A.p. and A. ervi-M.c. throughout this paper. Similarly, $A$. rhopalosiphi-S.a. and $A$. rhopalosiphi-M.d. will denote parasitoids reared from $S$. avenae and Metopolophium dirhodum, respectively.

\section{Host transfer trials}

Parasitoid performance was measured in terms of mummy production in standard pot trials. All trials were done using plastic plant pots $(13 \mathrm{~cm}$ in diameter $)$ covered by $17-\mathrm{cm}-$ tall, ventilated, clear plastic propagator covers. Pots used for trials with $A$. ervi contained either 40 (instars 2-4) individuals of Acyrthosiphon pisum on a four-leaved broad bean seedling or 40 (instars 2-4) individuals of Microlophium carnosum on a four-leaved nettle cutting. In trials with Aphidius rhopalosiphi, either 40 (instars 2-4) individuals or $0.02 \mathrm{~g}$ (all ages), which provided 60-80 aphids, of either $S$. avenae or Metopolophium dirhodum were placed on 20 two-leaved wheat seedlings in each pot. In all cases, the aphids were allowed to settle over a period of $24 \mathrm{~h}$. In one trial, the parasitoids were given a choice of hosts, each $A$. ervi pot containing 20 individuals of Acyrthosiphon pisum on a broad bean seedling plus 20 of Microlophium carnosum on a nettle cutting whilst each Aphidius rhopalosiphi pot contained 20 individuals of $S$. avenae plus 20 of Metopolophium dirhodum on 20 wheat seedlings.

Prior to their use in the trials, newly-emerged female parasitoids were placed in glass petri dishes $(5 \mathrm{~cm}$ in diameter) together with male parasitoids, water and dilute honey 
solution for $24 \mathrm{~h}$. Usually, two parasitoids of each sex were placed in each dish. After being allowed access to males and food, the female parasitoids were released into the trial pots, at one female per pot, and removed again after $24 \mathrm{~h}$. The pots were then kept at $20 \pm 1^{\circ} \mathrm{C}$ for $10-12$ days, and all the mummies that developed in each pot were counted and placed individually in small glass tubes until adult emergence. Emerging adults were sexed and either discarded or used in further trials. In all trials, ten replicate pots were set up, but pots were subsequently discarded if the female parasitoid was not recovered alive and apparently in good condition at the end of her 24 -h period in the pot. All the trials set up to measure mummy production are listed in Table I.

Parasitoid performance was also measured in terms of attack rate. Individual female parasitoids, fed and mated as described above, were released into small glass petri dishes ( $5 \mathrm{~cm}$ in diameter) containing 20 aphids in instars $2-4$ on leaves of the appropriate food-

\section{TABLE I. Trials set up to measure mummy production}

$\begin{array}{cl}\text { Trial } & \text { Parasitoid } \\ 1 & \text { Aphidius ervi } \\ 2 & \text { Aphidius ervi } \\ 3 & \text { Aphidius ervi } \\ 4 & \text { Aphidius ervi } \\ 5 & \text { Aphidius ervi } \\ 6 & \text { Aphidius ervi } \\ 7 & \text { Aphidius ervi } \\ 8 & \text { Aphidius ervi } \\ 9 & \text { Aphidius ervi } \\ 10 & \text { Aphidius ervi } \\ 11 & \text { Aphidius ervi } \\ & \\ 12 & \text { Aphidius ervi } \\ & \\ 13 & \text { Aphidius rhopalosiphi } \\ 14 & \text { Aphidius rhopalosiphi } \\ 15 & \text { Aphidius rhopalosiphi } \\ 16 & \text { Aphidius rhopalosiphi } \\ 17 & \text { Aphidius rhopalosiphi } \\ 18 & \text { Aphidius rhopalosiphi } \\ 19 & \text { Aphidius rhopalosiphi } \\ 20 & \text { Aphidius rhopalosiphi } \\ 21 & \text { Aphidius rhopalosiphi } \\ 22 & \text { Aphidius rhopalosiphi } \\ 23 & \text { Aphidius rhopalosiphi } \\ & \\ 24 & \text { Aphidius rhopalosiphi } \\ & \end{array}$

Origin of parasitoid
Lab. culture- $A$. pisum
Lab. culture- $A$. pisum
Lab. culture-M. carnosum
Lab. culture-M. carnosum
Lab. culture- $M$. carnosum
Ficld-collccted $A$. pisum
Ficld-collected $A$. pisum
Field-collected $M$. carnosum
Field-collected $M$. carnosum
Field-collected $M$. carnosum
F, offspring of crosses between parasitoids
from the two lab. cultures
F, offspring of crosses between parasitoids
from the two lab. cultures
Lab. culture- $S$. avenae
Lab. culture- $S$. avenae
Lab. culture- $S$. avenae
Lab. culture- $M$. dirhodum
Lab. culture- $M$. dirhodum
Lab. culture- $M$. dirhodum
Field-collccted $S$. avenae
Field-collected $S$. avenae
Field-collected $M$. dirhodum
Field-collected $M$. dirhodum
$F_{1}$ offspring of crosses between parasitoids
from the two lab. cultures
F, offspring of crosses between parasitoids
from the two lab. cultures

Test aphids

A. pisum

M. carnosum

M. carnosum

A. pisum

M. carnosum \& A. pisum

A. pisum

M. carnosum

M. carnosum

A. pisum

$S$. avenae

A. pisum

M. carnosum

S. avenae

$M$. dirhodum

$S$. avenae \& $M$. dirhodum

M. dirhodum

$S$. avenae

$S$. avenae \& $M$. dirhodum

$S$. avenae

$M$. dirhodum

$M$. dirhodum

$S$. avenae

S. avenae

M. dirhodum

plant. Each parasitoid was observed for a period of $30 \mathrm{~min}$, and the number of oviposition stabs which made full contact with an aphid was counted. All observations were made between 14.00 and $15.30 \mathrm{~h}$ to avoid diurnal influences on oviposition behaviour. The attack rate trials are listed in Table II. Ten parasitoids were used in each trial.

\section{TABLE II. Trials set up to measure attack rates}

$\begin{array}{cl}\text { Trial } & \text { Parasitoid } \\ 1 & \text { Aphidius ervi } \\ 2 & \text { Aphidius ervi } \\ 3 & \text { Aphidius ervi } \\ 4 & \text { Aphidius ervi } \\ 5 & \text { Aphidius rhopalosiphi } \\ 6 & \text { Aphidius rhopalosiphi } \\ 7 & \text { Aphidius rhopalosiphi } \\ 8 & \text { Aphidius rhopalosiphi }\end{array}$

Origin of parasitoid
Lab. culture- $A$. pisum
Lab. culture- $A$. pisum
Lab. culture- $M$. carnosum
Lab. culture- $M$. carnosum
Lab. culture- - avenae
Lab. culture- $S$. avenae
Lab. culture- $M$. dirhodum
Lab. culture- $M$. dirhodum

Test aphids

A. pisum

M. carnosum

M. carnosum

A. pisum

S. avenae

M. dirhodum

$M$. dirhodum

S. avenae

\section{Results}

\section{Aphidius ervi}

A. ervi-A.p. females from the laboratory culture which originated from, and was 
maintained on, Acyrthosiphon pisum produced very few mummies when transferred to Microlophium carnosum (Table III). In contrast, Aphidius ervi-M.c. females from the culture which originated from, and was maintained on, $M$. carnosum performed just as well when transferred to Acyrthosiphon pisum as they did on their original host. Similar results were obtained when parasitoids reared from mummies collected from field popula-

TABLE III. Mean ( \pm s.e.) number of mummies produced per female per $24 \mathrm{~h}$ in trials using Aphidius ervi

Origin of parasitoid

Lab. culture- $A$. pisum

Lab. culture-M. carnosum

Field-collected-A. pisum

Field-collected-M. carnosum

A. pisum
$28 \cdot 6 \pm 2 \cdot 7(7)^{*}$
$18 \cdot 2 \pm 3 \cdot 8(9)$
$19 \cdot 7 \pm 2 \cdot 0(10)$
$26 \cdot 3 \pm 1 \cdot 1(8)$

* The number of replicate pots is given in brackets. - $=$ Not tested.
Test aphids

$M$. carnosum
$1 \cdot 3 \pm 0 \cdot 5(9)$
$17 \cdot 6 \pm 1 \cdot 2(7)$
$1 \cdot 2 \pm 0 \cdot 5(10)$
$27 \cdot 3 \pm 1 \cdot 3(7)$

tions of these two host species were tested in the same way. Aphidius ervi-M.c. reared from field-collected mummies of $M$. carnosum were also transferred to $S$. avenae, but only two mummies in total were produced in the seven replicate pots. In a trial with both host species present (set up to test whether or not $A$. ervi-M.c. would still attack Acyrthosiphon pisum when its original host was also present), mummy production was the same for both hosts (Table III).

In the attack rate trials (Table IV), the greatest number of oviposition stabs was made by Aphidius ervi-A.p. attacking Acyrthosiphon pisum, and the lowest attack rate was recorded for Aphidius ervi-A.p. attacking $M$. carnosum. A. ervi-M.c. attacked $M$. carnosum and Acyrthosiphon pisum at similar rates. Attack rate was closely correlated with the mummy production observed in the previous trials.

TABle IV. Mean ( \pm s.e.) number of mummies produced per female per $24 \mathrm{~h}$ compared with the mean number of oviposition stabs per female per

30 min in trials using Aphidius ervi

Origin of parasitoid

Lab. culturc-A. pisum

Lab. culture-M. carnosum

Lab. culture $-M$. carnosum

Lab. culture--A. pisum

Test aphid
A. pisum
A. pisum
M. carnosum
M. carnosum

Mean no. of mummies

$$
\begin{array}{r}
28.6 \pm 2.7 \\
18.2 \pm 3.8 \\
17.6 \pm 1.2 \\
1.7 \pm 0.5
\end{array}
$$

Mean no. of oviposition stabs

$25 \cdot 0 \pm 3 \cdot 2$

$8 \cdot 6 \pm 1 \cdot 8$

$9.7+1.8$

$2.4 \pm(0.8$

Aphidiine parasitoids are arrhenotokous, which means that fertilized eggs give rise to diploid females and unfertilized eggs to haploid males. Thus, the production of female offspring indicates that the parent female has successfully mated. On this basis, successful mating was much more frequent in the case of Aphidius ervi females confined for $24 \mathrm{~h}$ with males from the same host species than for females confined with males which had emerged from a different host (Table V). Some of the female offspring from successful cross-

TABLE V. The percentage of successful matings (as indicated by the subsequent production of female offspring) between Aphidius ervi cultured on the same or different host species

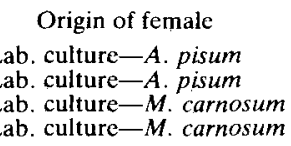

$$
\begin{aligned}
& \quad \text { Origin of male } \\
& \text { Lab. culture- } A \text {. pisum } \\
& \text { Lab. culture-M. carnosum } \\
& \text { Lab. culture-M. }- \text { carnosum } \\
& \text { Lab. culture- }- \text {. pisum }
\end{aligned}
$$

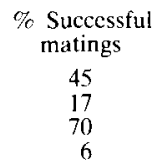

matings were used in mummy production trials. In all cases, the offspring had been reared 
on the same host species as their female parent. Although in the earlier trials (Table III) individual $A$. ervi-A.p. females had never produced more than four mummies when confined with $M$. carnosum, some offspring of the crosses in which the male parent had been reared on $M$. carnosum produced large numbers of mummies on that host, even though they themselves had been reared on Acyrthosiphon pisum (Table VI).

TABLE VI. Number of mummies produced by individual female offspring from matings between Aphidius ervi adults cultured on different host species*

\begin{tabular}{|c|c|c|c|c|}
\hline \multirow[b]{2}{*}{ Origin of femalc parent } & \multirow[b]{2}{*}{ Origin of male parent } & \multirow{2}{*}{$\begin{array}{c}\text { Female } \\
\text { offspring no. }\end{array}$} & \multicolumn{2}{|c|}{ Test aphid } \\
\hline & & & A. pisum & M. carnosum \\
\hline Lab. culture-A. pisum & Lab. culture-M. carnosum & $\begin{array}{l}1 \\
2 \\
3 \\
4\end{array}$ & $\begin{array}{l}31 \\
24 \\
- \\
-\end{array}$ & $\begin{array}{l}-\frac{}{27} \\
39\end{array}$ \\
\hline Lab. culture-A. pisum & Lab. culture-M. carnosum & $\begin{array}{l}1 \\
2 \\
3 \\
4\end{array}$ & $\begin{array}{l}18 \\
24 \\
- \\
-\end{array}$ & $\begin{array}{l}\overline{-} \\
\overline{14} \\
14\end{array}$ \\
\hline Lab. culture-A. pisum & Lab. culture- $M$. carnosum & $\begin{array}{l}1 \\
2 \\
3 \\
4\end{array}$ & $\begin{array}{r}18 \\
0 \\
- \\
-\end{array}$ & $\begin{array}{c}- \\
0 \\
2\end{array}$ \\
\hline Lab. culture-M. carnosum & Lab. culture-A. pisum & $\begin{array}{l}1 \\
2 \\
3 \\
4\end{array}$ & $\frac{-}{5}$ & $\begin{array}{r}3 \\
21 \\
- \\
-\end{array}$ \\
\hline
\end{tabular}
$24 \mathrm{~h}$.

* The offspring were reared on the same host species as the female parent and released onto 40 test aphids for

\section{Aphidius rhopalosiphi}

In the mummy production trials (Table VII), A. rhopalosiphi-M.d. produced significantly fewer mummies when transferred to $S$. avenae than when retained on Metopolophium dirhodum. In contrast, $A$. rhopalosiphi-S.a. showed an increase in mummy production when transferred to $M$. dirhodum. Thus, $A$. rhopalosiphi from the laboratory cultures always performed better on $M$. dirhodum than on $S$. avenae, regardless of the original host. When given a choice of hosts, $A$. rhopalosiphi-M.d. produced very few $S$. avenae mummies but $A$. rhopalosiphi-S.a. showed no apparent preference. In contrast, parasitoids reared from field-collected mummies performed equally well on both hosts, regardless of their original host. On average, laboratory-reared individuals produced more mummies $(51 \cdot 1)$ than did field-collected individuals $(33 \cdot 7)$ in trials with $M$. dirhodum $(t=2 \cdot 79, P<0 \cdot 01)$, but in trials with $S$. avenae laboratory-reared parasitoids produced fewer mummies (17.8) than did those from the field (32.0) $(t=3 \cdot 18, P<0 \cdot 01)$.

In the attack rate trials (Table VIII), again using females from the two laboratory cultures, both $A$. rhopalosiphi-M.d. and $A$. rhopalosiphi-S.a. attacked $M$. dirhodum at a greater rate than they attacked $S$. avenae. As was the case with $A$. ervi, these results reflect those obtained in the mummy production trials.

TABLE VIII. Mean ( \pm s.e.) number of mummies produced per female per $24 h$ compared with the mean number of oviposition stabs per female per 30 min in trials using Aphidius rhopalosiphi

\begin{tabular}{lllr}
\multicolumn{1}{c}{ Origin of parasitoid } & Test aphids & Mean no. of mummies & $\begin{array}{c}\text { Mean no. of } \\
\text { oviposition stabs }\end{array}$ \\
Lab. culture- $S$. avenae & M. dirhodum & $25 \cdot 6 \pm 2 \cdot 7 ; 44 \cdot 0 \pm 6 \cdot 1$ & $48 \cdot 3 \pm 1 \cdot 5$ \\
Lab. culture- $M$. dirhodum & M. dirhodum & $21 \cdot 3 \pm 4 \cdot 2 ; 58 \cdot 1 \pm 6 \cdot 1$ & $39 \cdot 7 \pm 5 \cdot 7$ \\
Lab. culturc- $S$. avenae & $S$. avenae & $11 \cdot 5 \pm 1 \cdot 9 ; 18 \cdot 1 \pm 2 \cdot 6$ & $24 \cdot 6 \pm 7.0$ \\
Lab. culture- $M$. dirhodum & $S$. avenae & $3 \cdot 5 \pm 0 \cdot 7 ; 17 \cdot 6 \pm 4 \cdot 8$ & $22 \cdot 0 \pm 3 \cdot 1$
\end{tabular}

${ }^{a} 40$ nymphs; $0 \cdot(2 \mathrm{~g}$ of aphids per trial pot. 


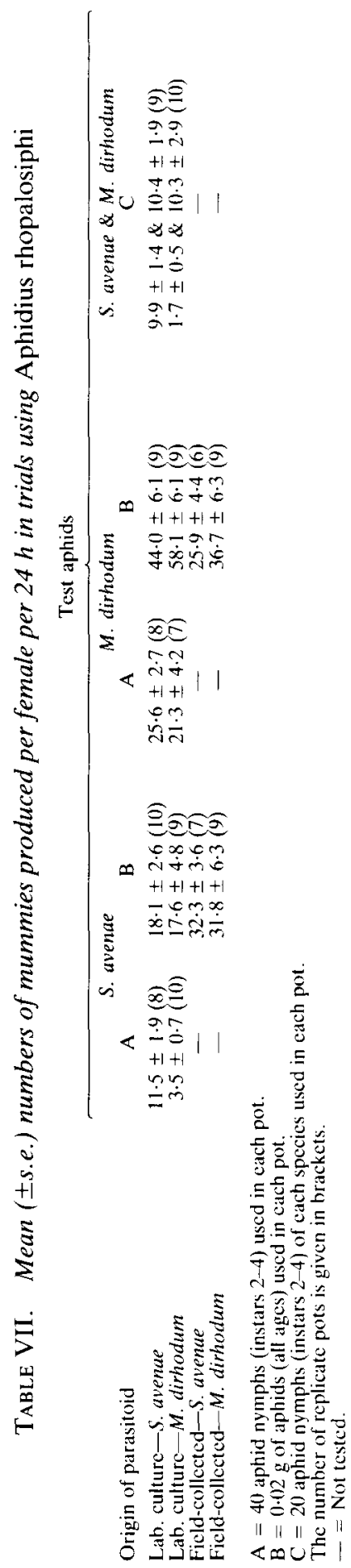


Unlike $A$. ervi, females of $A$. rhopalosiphi were as successful at mating with males which had been reared on the alternative host as they were with males originating from the

TABLE IX. The percentage of successful matings (as indicated by the subsequent production of female offspring) between Aphidius rhopalosiphi adults cultured on the same or different host species

\section{Origin of female}

Lab. culture-S. avenae

Lab. culturc-S. avenae

Lab. culture-M. dirhodum

Lab. culture-M. dirhodum

\author{
Origin of male \\ Lab. cuiture-S. avenae \\ Lab. culture-M. dirhodum \\ Lab. culture- $M$. dirhodum \\ Lab. culturc-S. avenae
}

\% Successful
matings
38
59
49
41

same host species (Table IX). When the offspring of cross-matings between A. rhopalosiphi-M.d. and $A$. rhopalosiphi-S.a. were used in mummy production trials, variable results were obtained. In the first series of trials (Table X), each pot contained 40 aphid nymphs in instars $2-4$, and no more than three (in most cases two) offspring from each individual parasitoid pairing were tested on each aphid host. Examination of the results suggested there was a tendency for the offspring of any individual cross to prefer one of the two host species, but this was not always $M$. dirhodum as might have been expected from the results of earlier trials (Table VII). In the second series of trials (Table XI), each pot contained $0.02 \mathrm{~g}$ of aphids of mixed ages, and more offspring from each cross were tested on each host. The tendency for siblings to all perform better on one host than on the other was no

\section{TABLE X. Number of mummies produced by individual female offspring from} matings between Aphidius rhopalosiphi adults cultured on different host

$$
\text { species* }
$$

Origin of female parent

Lab. culture-M. dirhodum

Origin of malc parent Lab. culture-S. avenae

Lab. culture- $-M$. dirhodum Lab. culture $-S$. avenae

Lab. culture- $M$. dirhodum Lab. culture-S. avenae

Lab. culture--M. dirhodum Lab. culture-S. avenae

Lab. culture-S. avenae

Lab. culturc- $-M$. dirhodum

Lab. culture-S. avenae

Lab, culture--S. avenae

Lab. culturc-S. avenae

Lab. culturc- $M$. dirhodum

\begin{tabular}{|c|c|}
\hline \multicolumn{2}{|c|}{ Test aphids } \\
\hline S. avenae & M. dirhodum \\
\hline- & 2 \\
\hline- & 8 \\
\hline- & 1 \\
\hline 30 & - \\
\hline 34 & - \\
\hline- & 14 \\
\hline$\ldots$ & 32 \\
\hline 8 & - \\
\hline 10 & - \\
\hline - & 21 \\
\hline 9 & - \\
\hline 9 & - \\
\hline- & 23 \\
\hline$\ldots$ & 40 \\
\hline 2 & - \\
\hline 14 & - \\
\hline 7 & - \\
\hline 7 & - \\
\hline- & 38 \\
\hline - & 40 \\
\hline 7 & - \\
\hline 1 & - \\
\hline- & 39 \\
\hline- & 33 \\
\hline 27 & - \\
\hline 19 & - \\
\hline- & 3 \\
\hline 16 & - \\
\hline- & 0 \\
\hline- & 1 \\
\hline
\end{tabular}

\footnotetext{
* The offspring were reared on the same host species as the female parent and released onto 40 test aphids for
} $24 \mathrm{~h}$. 
TABLE XI. Mean number and range of mummies produced by female offspring from matings between Aphidius rhopalosiphi adults cultured on different host species*

\author{
Origin of female parent \\ Lab. culture- $M$. dirhodum \\ Lab. culture- $M$. dirhodum \\ Lab. culture-S. avenae
}

\author{
Origin of male parent \\ Lab. culture-S, avenae \\ Lab. culture-S. avenae \\ Lab. culturc-M. dirhodum
}

\begin{tabular}{cc}
\multicolumn{2}{c}{ Test aphids } \\
S. avenae & M. dirhodum \\
$16 \cdot 3(7)$ & $63 \cdot 3(8)$ \\
$1-49$ & $43-93$ \\
$28.9(9)$ & $42 \cdot 5(8)$ \\
$21-42$ & $29-54$ \\
$33 \cdot 8(4)$ & $46 \cdot 5(4)$ \\
$16-46$ & $27-64$
\end{tabular}

* The offspring were reared on the same host as the female parent and released onto $0.02 \mathrm{~g}$ test aphid for $24 \mathrm{~h}$. The number of individuals tested is given in brackets.

longer apparent, and variability between individuals was very high, suggesting that the apparent host preferences noted in the first series were not real but a result of the low number of parasitoids tested.

\section{Discussion}

Although Aphidius ervi is commonly recorded from a number of different aphid hosts, attempts to transfer it from one host to another in laboratory trials have met with limited success (Table XII). In the present trials, it readily transferred from Microlophium

TABLE XII. The success of laboratory host transfer trials using Aphidius ervi and three of its major hosts, Microlophium carnosum, Acrythosiphon pisum and Sitobion avenae, as recorded in four separate studies

$\begin{array}{ll}\text { Origin of parasitoid } & \text { Test aphid } \\ \text { A. pisum } & \text { M. carnosum } \\ \text { A. pisum } & \text { M. carnosum } \\ \text { M. carnosum } & \text { A. pisum } \\ \text { M. carnosum } & \text { A. pisum } \\ \text { M. carnosum } & \text { A. pisum } \\ \text { M. carnosum } & \text { S. avenae } \\ M . \text { carnosum } & \text { S. avenae } \\ \text { A. pisum } & \text { S. avenae } \\ \text { A. pisum } & \text { S. avenae } \\ \text { S. avenae } & \text { A. pisum }\end{array}$

\begin{tabular}{cl} 
Exposure & \multicolumn{1}{c}{ Result } \\
time & Unsuccessful \\
$18 \mathrm{~h}$ & Very few mummies \\
$24 \mathrm{~h}$ & Very few mummies \\
$6 \mathrm{~h}$ & Very few mummies \\
$?$ & Successful \\
$24 \mathrm{~h}$ & Unsuccessful \\
$?$ & Very few mummies \\
$24 \mathrm{~h}$ & Successful after \\
$18 \mathrm{~h}$ & 4 generations \\
& Successful \\
$?$ & Unsuccessful
\end{tabular}

\[ \text { Source } \]
Cameron et al. (1984)
Present study
Starý et al. (1985)
Pungerl (1984)
Present study
Pungerl (1984)
Present study
Cameron et al. (1984)
Pungerl (1984)
Pungerl (1984)

carnosum to Acyrthosiphon pisum, without any apparent reduction in performance (measured as mummy production), but it prduced very few mummies when transferred from $A$. pisum to $M$. carnosum. However, in other trials, Aphidius ervi produced very few mummies when transferred from M. carnosum to Acyrthosiphon pisum (Pungerl, 1984; Starý et al., 1985). Furthermore, Pungerl (1984) successfully transferred Aphidius ervi from Acyrthosiphon pisum to $S$. avenae, as did Cameron et al. (1984), but Pungerl's efforts to transfer it in the opposite direction failed.

Similar inconsistencies occurred when Aphidius rhopalosiphi was transferred between $S$. avenae and another cereal aphid, Metopolophium dirhodum. Parasitoids from our laboratory cultures always performed well on $M$. dirhodum, regardless of their original host, but did less well on $S$. avenae, particularly when transferred from $M$. dirhodum. In contrast, Ankersmit (1983) found that $A$. rhopalosiphi cultured on $S$. avenae performed very poorly when transferred to $M$. dirhodum.

How can these inconsistent results be explained? It is significant that most of these transfer trials were done using laboratory cultures. Laboratory populations of parasitoids are known to suffer rapid reductions in genetic diversity as a result of genetic drift (Unruh et al., 1983). Our laboratory cultures were started from small founder populations collected in the field, and Pungerl (1984) used single mated females to initiate her cultures. If 
genetics plays a significant role in determining the ability of a parasitoid to transfer from one host species to another, then the performances of different laboratory populations could indeed vary.

There are several indications that genetics does play a role in determining the success of host transfers by aphid parasitoids. Němec \& Starý (1983) reared $A$. ervi from fieldcollected individuals of Microlophium carnosum and then confined them with Acyrthosiphon pisum in the laboratory. Although very few mummies were produced in the first generation, continued selection of these led to the development of a laboratory strain of the parasitoid which successfully utilized the new host. Electrophoretic studies revealed that this strain was monomorphic for those enzyme systems tested, whereas the original field population on $M$. carnosum was much more genetically diverse. Similarly, when Aphidius ervi was transferred from Acyrthosiphon pisum to $S$. avenae, mummy production was greatly reduced in the first generation but subsequently recovered over several generations on the new host (Cameron et al., 1984). Again, analysis of esterase allozymes using electrophoresis suggested that a strain of Aphidius ervi had been selected on $S$. avenae from a more diverse genotype on the original host. The importance of genotype has been further emphasized in the present study. Mummy production by $A$. ervi was very poor when it was transferred from Acyrthosiphon pisum to $M$. carnosum. However, this was often dramatically improved by mating females of Aphidius ervi-A.p. with males of $A$. ervi-M.c. The female offspring from these crosses, even though they themselves were reared on Acyrthosiphon pisum, readily transferred to $M$. carnosum, indicating that the male parent had provided genetic input which influenced their ability to transfer between hosts.

There is evidence that field populations of aphid parasitoids are generally genetically more diverse than are laboratory populations. Unruh et al. (1983) and Němec \& Starý (1985) demonstrated that field populations of Aphidius ervi had much greater enzyme variability, as measured by electrophoresis, than did laboratory cultures. Unruh et al. (1983) calculated that, under the normal culturing regime operated in their laboratory, heterozygosity of an $A$. ervi population would be halved in less than nine months in culture. This would explain why parasitoids from field populations sometimes perform differently in host-transfer trials to parasitoids from laboratory cultures, as in the case of $A$. rhopalosiphi in the present trials. It is perhaps not surprising, therefore, that different laboratory populations, especially those originating from different field populations, often vary in their behavioural and biological characteristics.

Poor mummy production following host transfer could be the result of a reduced attack rate on the new host or of more efficient physiological defence reactions by the new host, preventing parasitoid development. Here again, results from different studies are inconsistent. Starý et al. (1985) recorded high attack rates by $A$. ervi after transfer from $M$. carnosum to Acyrthosiphon pisum, but this was followed by poor mummy production. They concluded that host defence mechanisms were the main cause of unsuccessful host transfers. Ankersmit (1983) could not detect any change in attack rate when Aphidius rhopalosiphi was transferred from $S$. avenae to Metopolophium dirhodum and so he also attributed poor mummy production to internal defences. In the present trials, however, the results suggest that poor mummy production was partly the result of low attack rates, at least in the case of $A$. ervi. There is no reason, of course, why both factors should not operate.

Laboratory host-transfer trials can only answer the question: is the parasitoid capable of transferring from one host species to another? Furthermore, it seems that to answer this question adequately it is necessary to do a series of trials, each time using fresh laboratory populations, preferably each originating from a different field population. Based on current evidence, the ability or willingness to transfer between hosts is likely to vary between different laboratory populations and will not necessarily reflect the situation in wild populations. Once it has been established that a parasitoid species is capable of transferring between two or more host species, a more important question is: does the parasitoid transfer between these hosts in the field? The fact that the same parasitoid species can be 
reared from field populations of more than one host species does not prove that there is any exchange of individuals between them.

Electrophoretic analyses of field populations of $A$. ervi on different hosts (Němec \& Starý, 1983) and morphological studies of their colour patterns (Starý, 1983b) led to the conclusion that the parasitoid attacking Microlophium carnosum is a distinct biotype, which does not move between this host and its other main hosts on nearby arable crops, but that transfers do occur between $S$. avenae and Acyrthosiphon pisum in the field. The low percentage of successful matings between Aphidius ervi-M.c. and $A$. ervi-A.p. noted in the present trials tends to support this view. Nevertheless, in these trials, both laboratoryreared and field-collected individuals of $A$. ervi readily transferred from $M$. carnosum to Acyrthosiphon pisum. It is obvious, therefore, that more work is urgently needed on the population dynamics of parasitoids in the field and that comparisons of populations from different geographical regions may prove enlightening.

The variation in behavioural and biological characteristics between different laboratory cultures of the same parasitoid species and the genetic variability that can be present in wild populations raises the question of selective breeding. Results of the host transfer trials point to the possibility of selecting for the ability to transfer between designated hosts or for improved performances on one specific host. However, there may be problems with the ability of selectively-bred laboratory strains to become permanently established in the field. Selection for a particular characteristic could involve concomitant selection for less desirable traits which may reduce fitness in the wild and the parasitoids' ability to compete with existing wild populations. Nevertheless, selected strains may prove invaluable for inundative releases aimed at enhancing parasitoid impact on pest populations at critical times in their development. The present trials indicate the importance of genotype in determining the ability of Aphidius ervi to transfer between hosts. If genotype influences the response of the parasitoid to semiochemicals during host searching and attack behaviour, then there could be potential for selective breeding to aid the use of behaviourcontrolling chemicals to manipulate parasitoid activity in the field. Successful selection for specific characteristics has been achieved with other parasitoids (Wilkes, 1947; White et al., 1970), and the potential of selective breeding of parasitoids in biological control has been discussed by several authors (DeBach, 1958; Simmonds, 1963; Mackauer, 1972, 1976).

If efficient use is to be made of alternative hosts to enhance parasitoid impact on insect pests, it must be based on a thorough understanding of the population dynamics of the parasitoid in the field. We know very little about the movements of parasitoids within agroecosystems and about how and when they utilize different hosts and other resources in both crops and semi-natural habitats. The potential of $M$. carnosum as a reservoir host for $A$. ervi remains problematical, awaiting more extensive field data on the parasitoid's population dynamics in arable farmland.

\section{Acknowledgements}

The Environmental Research Fund is acknowledged for their financial support of part of this work.

\section{References}

ANKERSMit, G. W. (1983). Aphidiids as parasites of the cereal aphids Sitobion avenae and Metopolophium dirhodum. - pp. 42-49 in Cavalloro, R. (Ed.). Aphid antagonists.-143 pp. Rotterdam, Balkema.

Cameron, P. J., Powell, W. \& Loxdale, H. D. (1984). Reservoirs for Aphidius ervi Haliday (Hymenoptera: Aphidiidae), a polyphagous parasitoid of cereal aphids (Hemiptera: Aphididae).-Bull. ent. Res. 74, 647-656.

DeBach, P. (1958). Selective breeding to improve adaptations of parasitic insects.-Proc. loth Int. Congr. Ent. 4, 759-768.

MackaUer, M. (1972). Genetic aspects of insect production,-Entomophaga 17, 27-48.

MACKAUER, M. (1976). Genetic problems in the production of biological control agents.-A. Rev. Ent. 21, 369-385. 
Němec, V. \& Starý, P. (1983). Elpho-morph differentiation in Aphidius ervi Hal. biotype on Microlophium carnosum (Bckt.) related to parasitization on Acyrthosiphon pisum (Harr.) (Hym., Aphidiidae).-Z. angew. Ent. 95, 524-530.

Němec, V. \& Starý, P. (1985). Genetic diversity of the parasitoid Aphidius ervi on the pea aphid, Acyrthosiphon pisum in Czechoslovakia (Hymenoptera, Aphidiidae; Homoptera, Aphididae).-Acta entomol. bohemoslov. 82, 88-94.

Nordlund, D. A. (1987). Plant produced allelochemics and their involvement in the host selection behaviour of parasitoids.--pp. 103-107 in Labeyrie, V., Fabres, G. \& Lachaise, D. (Eds). Insects-plants.- 459 pp. Dordrecht, Junk.

PERrin, R. M. (1975). The role of the perennial stinging nettle, Urtica dioica, as a reservoir of beneficial natural enemies.-Ann. appl. Biol. 81, 289-297.

Powell, W. (1986). Enhancing parasitoid activity in crops.-pp. 319-340 in Waage, J. \& Greathead, D. (Eds). Insect parasitoids.-389 pp. London, Academic Press (Symp. R. Ent. Soc. Lond. no. 13).

Powell, W. \& Zhang, Z. L. (1983). The reactions of two cereal aphid parasitoids, Aphidius uzbekistanicus and $A$. ervi to host aphids and their food-plants.-Physiol. Entomol. 8, 439 443.

Pungerl, N. B. (1984). Host preferences of Aphidius (Hymenoptera: Aphidiidae) populations parasitising pea and cereal aphids (Hemiptera: Aphididae).-Bull. ent. Res. 74, 153-161.

READ, D. P., FEENY, P. P. \& RoOT, R. B. (1970). Habitat selection by the aphid parasite Diaeretiella rapae (Hymenoptera: Braconidae) and hyperparasite Charips brassicae (Hymenoptera: Cynipidae).-Can. Ent. 102, 1567-1578.

Simmonds, F. J. (1963). Genetics and biological control.-Can. Ent. 95, 561-567.

StaRÝ, P. (1983a). The perennial stinging nettle (Urtica dioica) as a reservoir of aphid parasitoids (Hymenoptera, Aphidiidae).-Acta entomol bohemoslov. 80, 81-86.

Starý, P. (1983b). Colour patterns of adults as evidence on Aphidius ervi biotypes in field environments (Hymenoptera. Aphidiidae). - Acta entomol. bohemoslov. 80, 377-384.

StarÝ, P. (1986). Creeping thistle, Cirsium arvense, as a reservoir of aphid parasitoids (Hymenoptera, Aphidiidae) in agroecosystems. - Acta entomol. bohemoslov. 83, 425-431.

StaRÝ, P. \& NĚmec, V. (1986). Common elder, Sambucus nigra, as a reservoir of aphids and parasitoids (Hymenoptera, Aphidiidae),-Acta entomol. bohemoslov. 83, 271-278.

Starý, P., Pospíśll, J. \& NĚMEc, V. (1985). Integration of olfactometry and electrophoresis in the analysis of aphid parasitoid biotypes (Hym., Aphidiidae).-Z. angew. Ent. 99, 476-482.

Unruh, T. R., White, W., González, D., Gordh, G. \& Luck, R. F. (1983). Heterozygosity and effective size in laboratory populations of Aphidius ervi (Hym.: Aphidiidae).-Entomophaga 28, 245-258.

VAN DEN BOSCH, R. \& TELFORD, A. D. (1964). Environmental modification and biological control.pp. 459-488 in DeBach, P. (Ed.). Biological control of insect pests and weeds. -844 pp. London, Chapman \& Hall.

White, E. B., DeBach, P. \& Garber, M. J. (1970). Artificial selection for genetic adaptation to temperature extremes in Aphytis lingnanensis Compere (Hymenoptera: Aphelinidae).Hilgardia 40, 161-192.

WILKES, A. (1947). The effects of selective breeding on the laboratory propagation of insect parasites.-Proc. R. Soc. (B) 134, 227-245.

(Received 19 Apil 1988)

(C) C.A.B International, 1988 